\title{
INTEGRAÇÃO DA PRODUÇÃO DE FURFURAL EM UMA BIORREFINARIA DE CANA-DE-AÇÚCAR
}

\author{
J. F. L. SILVA ${ }^{1,2}$, M. A. SELICANI ${ }^{1}$, T. L. JUNQUEIRA ${ }^{1}$, B. C. $\operatorname{KLEIN~}^{1,2}$, A. BONOMI ${ }^{1,2}$ \\ ${ }^{1}$ CTBE/CNPEM - Laboratório Nacional de Ciência e Tecnologia do Bioetanol \\ ${ }^{2}$ Universidade Estadual de Campinas, Faculdade de Engenharia Química \\ E-mail para contato: jean.silva@bioetanol.org.br
}

\begin{abstract}
RESUMO - Furfural é um químico de base com variadas aplicações e com grande expectativa de crescimento de mercado. Derivado de biomassa, sua produção pode ser incorporada à indústria brasileira através do uso de bagaço de cana-deaçúcar, usualmente destinado à geração de energia (vapor e eletricidade). Neste contexto, a integração de uma planta de furfural - utilizando parte do bagaço disponível como matéria-prima - à produção de etanol no cenário brasileiro foi simulada e comparada a uma destilaria de etanol. Os resultados mostraram que a planta integrada tem uma taxa interna de retorno de $16,7 \%$, que é 4 p.p. superior à da destilaria autônoma. No contexto da biorrefinaria de cana-de-açúcar, a produção de furfural é vantajosa por ser um produto de maior valor agregado e investimento relativamente baixo.
\end{abstract}

\section{INTRODUÇÃO}

Hoje, compostos químicos de origem vegetal estão ganhando considerável espaço no mercado graças ao ideal de criação de uma indústria química baseada em bioderivados e também devido à pressão política. Esta tendência vem da oscilação dos preços do petróleo, da busca por fontes de energia alternativas e da visão de garantir um suprimento energético renovável. O conceito de biorrefinaria ganha força para suportar esta necessidade (De Jong e Marcotullio, 2010; Moulijn et at., 2012). Bioetanol, o mais conhecido bioderivado, tem um mercado consolidado no mundo e uma situação de sucesso no mercado brasileiro. Muita atenção é dada à destinação do bagaço e da palha que hoje são usados como combustível em unidades de cogeração (produção e utilização combinada de vapor e eletricidade) e possuem potencial aplicação na produção de etanol de segunda geração ou celulósico (Mariano et al., 2013). Também, muito esforço é destinado à criação de novas rotas de utilização da biomassa para produzir compostos de maior valor agregado.

Da mesma maneira que a indústria petroquímica baseia-se em cerca de 20 compostos derivados de petróleo (Moulijn et at., 2012), é possível imaginar uma série de compostos derivados de biomassa para aplicações gerais na indústria química de base. Um destes compostos é o furfural, reconhecido como uma plataforma química para uma ampla gama de aplicações (De Jong e Marcotullio, 2010). Usos atuais consistem na derivação de furanos e uso como solvente em processos extrativos. Sua produção é consagrada na indústria através da hidrólise de biomassa rica em pentoses e a consequente desidratação do açúcar formado. A 
hidrólise da biomassa é em geral conduzida em meio ácido, e a quantidade de processos existentes é ampla e varia com a matéria-prima (Zeitsch, 2000). A demanda de furfural em 2013 foi de $300 \mathrm{kt} / \mathrm{a}$, e espera-se que será de $652 \mathrm{kt} / \mathrm{a}$ em 2020 (GVR, 2015).

O processo Rosenlew consiste em um digestor de bagaço de cana-de-açúcar com a vantagem de ser um processo autocatalítico. $O$ uso desta tecnologia é consagrado no ambiente industrial e é empregado na segunda maior planta de produção de furfural do mundo, situada na África do Sul (Zeitsch, 2000). Porém, a maior parte do furfural produzido no mundo vem da China, obtido através do Processo em Batelada Chinês, que utiliza sabugo de milho como matéria-prima. Devido à disponibilidade de bagaço de cana-de-açúcar no mercado brasileiro, é interessante considerar a incorporação de uma planta de produção de furfural a uma destilaria de etanol de primeira geração.

O objetivo deste trabalho é de avaliar os impactos econômicos da integração de uma planta de produção de furfural a uma destilaria de etanol. A planta de furfural terá capacidade de produção de $21 \mathrm{kt} / \mathrm{a}$, equivalente a $6 \%$ do mercado mundial em 2014. A modificação consiste em integrar um processo já consagrado para usar matéria-prima de baixo custo e integrar o uso de utilidades, que é um dos fatores que mais influenciam no custo de produção do furfural.

\section{MÉTODOS}

\subsection{Descrição do processo}

O processo consiste em uma planta de furfural integrada a uma planta de produção etanol com capacidade de processamento anual de 4 milhões de toneladas de cana-de-açúcar (TC). A planta de etanol é projetada para operar durante 200 dias de safra, enquanto que a unidade de cogeração de calor e potência (CHP) e a unidade de produção de furfural operam 330 dias/ano. São processadas 833 t/h de cana-de-açúcar e, após extração do caldo, produz-se $221 \mathrm{t} / \mathrm{h}$ de bagaço. Deste bagaço, 5\% é estocado para inicialização da unidade de CHP. Do restante, $40 \%$ seguem para o consumo imediato no CHP durante a safra, $41 \%$ segue para a planta de furfural e o restante é estocado para alimentar a unidade de CHP na entressafra. Parte da biomassa que segue para a planta de furfural é estocada para operação na entressafra. Considera-se também o processamento de $68 \mathrm{t} / \mathrm{h}$ de palha de cana-de-açúcar (equivalente a $50 \%$ da produzida no campo), que seguem diretamente para a queima na caldeira em operação durante a safra. O caldo extraído segue para o processo de produção do etanol, através da fermentação dos açúcares, conhecido como processo de primeira geração (1G). A caldeira opera produzindo vapor a 65 bar, o que a leva a ter maior eficiência térmica do que as tradicionais caldeiras de 22 bar e proporciona a produção de eletricidade, usada na planta e vendida para a rede (Mariano et al., 2013).

Para a produção de furfural, o bagaço é inserido no digestor de Rosenlew, que opera a $180{ }^{\circ} \mathrm{C}$ e 10 bar. Vapor a 10 bar superaquecido $\left(265^{\circ} \mathrm{C}\right)$ entra no fundo do reator, promove a hidrólise da xilana e sua conversão a furfural, o qual é removido do reator por arraste a vapor. $\mathrm{O}$ resíduo deste reator segue para um vaso flash para recuperar furfural ainda presente na biomassa e após isso é destinado à queima, uma vez que o sólido possui capacidade calorífica significativa. A corrente de vapor, produto da reação, segue então para a sequência de destilação proposta por Zeitsch (2000). Água é removida como produto de fundo em uma 
primeira coluna. O topo desta coluna gera uma corrente com furfural, água e componentes voláteis que segue para uma segunda coluna onde o topo elimina os voláteis (metanol, etanol e diacetil principalmente), que são levados para queima na CHP. O fundo desta segunda coluna e uma corrente lateral da primeira coluna consistem majoritariamente de furfural e água em uma mistura azeotrópica. Estas correntes são resfriadas e destinadas a um decantador, onde duas fases são obtidas: 1) a aquosa, que retorna à primeira coluna e 2) a fase orgânica rica em furfural (acima de 90\%). A fase orgânica segue para uma terceira coluna, onde se obtém no fundo furfural com $98,5 \%$ de pureza. O topo - uma mistura azeotrópica de água e furfural - retorna ao decantador. Um esquema simplificado de todo este processo encontra-se na Figura 1.

Figura 1 - Fluxograma do processo de produção de furfural integrado ao de etanol.

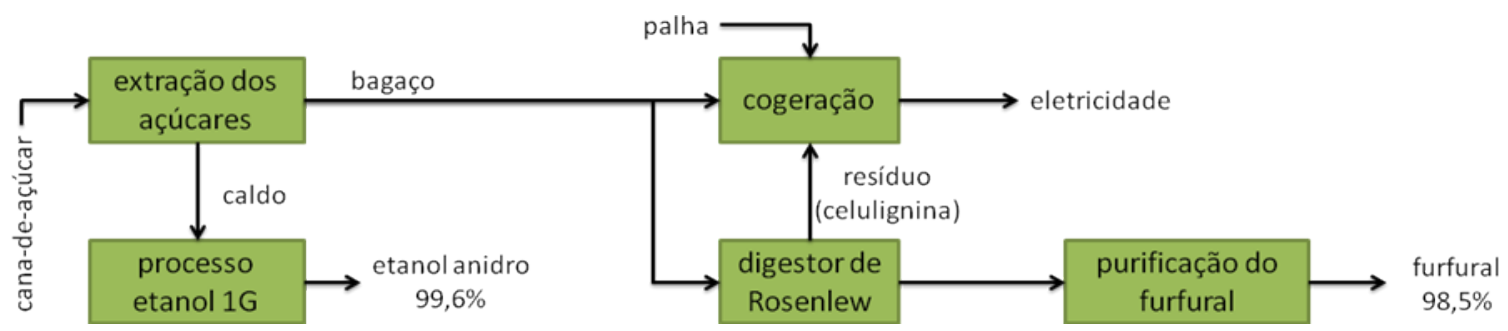

\subsection{Simulação do processo}

A biorrefinaria integrada de produção de etanol $1 \mathrm{G}$ e furfural foi simulada no Aspen Plus (versão 7.3.2). A configuração da planta e os parâmetros de processo utilizados para simulação da produção de etanol $1 \mathrm{G}$ e da etapa de cogeração são baseados na Biorrefinaria Virtual de Cana-de-Açúcar, ferramenta desenvolvida pelo Laboratório Nacional de Ciência e Tecnologia do Bioetanol (CTBE) (Dias et al., 2011; CTBE, 2012; Mariano et al., 2013). Adaptações no modelo de destilaria de etanol previamente desenvolvido foram realizadas para incluir o processo de produção de furfural, as diferenças entre os períodos de operação (safra e entressafra) e a integração do uso de utilidades (e.g. vapor). O digestor Rosenlew foi simulado como um reator RStoic para conversão e uma coluna RadFrac para a separação das fases líquida e vapor. As colunas usadas para a purificação do furfural são simuladas com o modelo RadFrac e com parâmetros apresentados por Zeitsch (2000). A Figura 2 apresenta o fluxograma simplificado do modelo feito no Aspen Plus.

Figura 2 - Fluxograma simplificado do processo de produção do furfural no Aspen Plus.

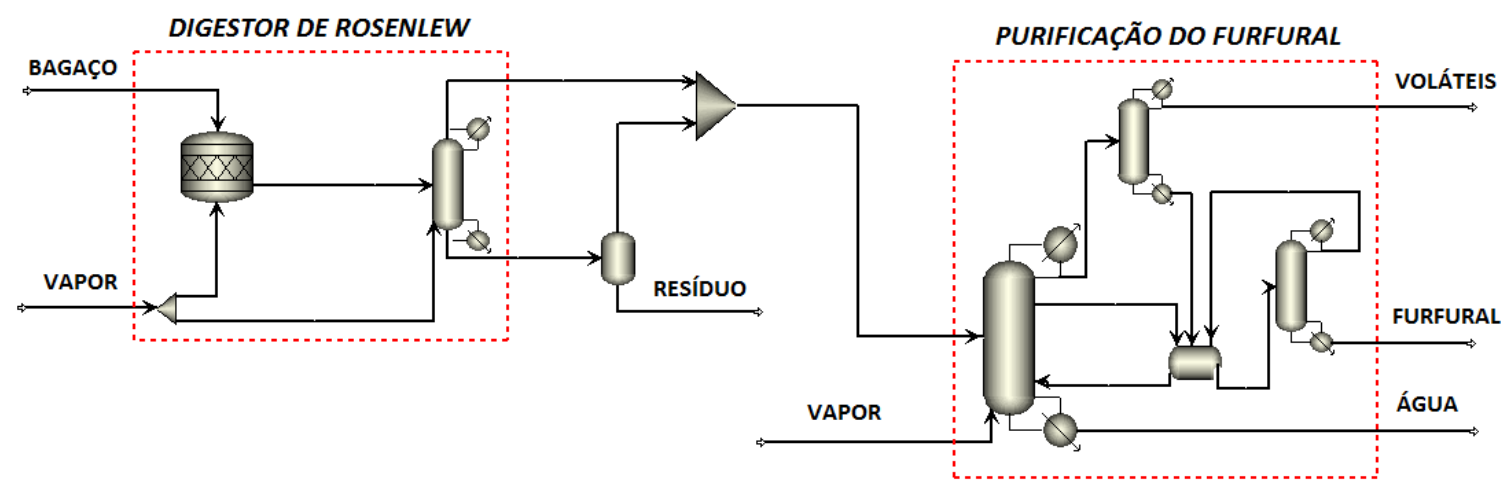




\subsection{Análise técnico-econômica}

Foram avaliados o investimento e o custo de produção para cada um dos seguintes cenários no contexto da indústria brasileira: 1) 1GBASE: base, correspondente à destilaria autônoma que consome todo o bagaço para produzir eletricidade como subproduto; e 2) $1 \mathrm{G}+\mathrm{FF}$ : correspondente à planta de furfural integrada à usina autônoma, utilizando parte do bagaço com matéria-prima. $\mathrm{O}$ investimento em equipamentos e a instalação foram estimados a partir da base de dados do CTBE e o capital de giro é considerado como $10 \%$ do investimento capital fixo. A análise econômica foi realizada com base nos dados da Tabela 1, considerando um tempo de vida de projeto de 25 anos, com tempo de construção e comissionamento de 2 anos, depreciação linear nos 10 primeiros anos e valor residual nulo.

Tabela 1 - Premissas para a análise econômica

\begin{tabular}{lll}
\hline Parâmetro & Valor & Referência \\
\hline Taxa mínima de atratividade - TMA $(\%)$ & 12,0 & \\
Alíquota de tributos $(\%)$ & 34,0 & \\
Preço da cana-de-açúcar $(\mathrm{R} \$ / \mathrm{t})$ & 66,95 & CanaSoft $^{1}$ \\
Preço da palha $(\mathrm{R} \$ / \mathrm{t})$ & 62,31 & CanaSoft $^{1}$ \\
Preço da eletricidade $(\mathrm{R} \$ \mathrm{MWh})$ & 132,43 & MME, média de leilões $(2005-2013)$ \\
Preço do etanol anidro $(\mathrm{R} \$ / \mathrm{L})$ & 1,34 & CEPEA, média móvel $(2004-2014)$ \\
Preço do furfural $(\mathrm{R} \$ / \mathrm{kg})$ & 3,28 & CEPEA, média móvel $(2004-2014)$ \\
\hline
\end{tabular}

${ }^{1}$ Modelo da fase agrícola integrado à Biorrefinaria Virtual de Cana-de-açúcar

\section{RESULTADOS E DISCUSSÃO}

\subsection{Análise técnico-econômica}

Os principais resultados obtidos na avaliação econômica são apresentados na Tabela 2. Destaca-se a receita anual com os produtos: um adicional de $\mathrm{R} \$ 69$ milhões relativo à venda de furfural comparado a uma redução de R\$ 29 milhões na receita com eletricidade, o que é uma perspectiva bastante promissora. Além do mais, a destinação de parte do bagaço para a planta de furfural no cenário $1 \mathrm{G}+\mathrm{FF}$ e maior período de operação $(330 \mathrm{~d} / \mathrm{a})$ diminuem a necessidade de capacidade na unidade de CHP. Esta diminuição na capacidade de processamento da unidade de CHP é mais do que suficiente para cobrir as despesas com capital total inicial da unidade de produção de furfural.

Um aspecto importante é o preço de mercado do furfural comparado ao preço da eletricidade, considerando que ambos podem ser produzidos a partir da mesma matéria-prima. Nos dois cenários analisados, esta comparação torna-se complicada, uma vez que, no cenário com furfural, parte da eletricidade e parte do vapor de processo são gerados a partir do resíduo do digestor de Rosenlew. Porém, observando o custo de produção alocado para a eletricidade e para o etanol na Tabela 2, nota-se uma redução de $11 \%$ em ambos, uma vez que neste novo cenário a planta conta com a receita adicional do furfural. Outro parâmetro importante para a análise comparativa de projetos é a taxa interna de retorno (TIR), que indica a rentabilidade de um empreendimento. Pela Tabela 2, pode-se constatar que a integração da produção de 
furfural a uma planta de etanol $1 \mathrm{G}$ favorece o retorno financeiro da biorrefinaria, com uma TIR superior em 4 p.p. em relação ao cenário base.

Tabela 2 - Resultados do estudo baseados na simulação e na análise econômica

\begin{tabular}{lll}
\hline \multirow{2}{*}{ Parâmetro } & Cenário \\
\cline { 2 - 3 } & 1GBASE & $1 \mathrm{G}+\mathrm{FF}$ \\
\hline Produção de etanol (kt/a) & 266 & 266 \\
Produção de eletricidade para a rede (MWh/a) & 773 & 555 \\
Produção de furfural (kt/a) & - & 21 \\
Investimento (R\$, milhões) & 1028,6 & 898,4 \\
Custo de produção de etanol (R\$/L) & 1,16 & 1,03 \\
Custo de produção da eletricidade (R\$/MWh) & 114,56 & 102,01 \\
Custo de produção do furfural (R\$/kg) & - & 2,53 \\
Taxa interna de retorno (\% a.a.) & 12,7 & 16,7 \\
Receita anual com etanol (R\$, milhões) & 450,5 & 450,5 \\
Receita anual com eletricidade (R\$, milhões) & 102,3 & 73,5 \\
Receita anual com furfural (R\$, milhões) & - & 69,0 \\
\hline
\end{tabular}

\subsection{Análise de sensibilidade}

Os resultados da análise econômica são bastante dependentes dos preços dos produtos no mercado e das incertezas sobre o investimento. Portanto, para analisar o impacto destes parâmetros sobre a lucratividade da planta, foi realizada uma análise de sensibilidade para a TIR como função de variações de $\pm 25 \%$ no capital total inicial da planta do cenário $1 \mathrm{G}+\mathrm{FF}$ e nos preços de cana, palha, etanol, eletricidade e furfural. Os resultados apresentados na Figura 3 mostram que, de fato, a TIR é mais sensível ao preço do principal produto da planta, o etanol. Porém, o resultado mais notável é que a única situação em que a planta deixa de ser um investimento atrativo (TIR<TMA) é para uma queda no preço do etanol maior que $18 \%$. Variações no preço do furfural, mercado que crescerá muito nos próximos anos, não representam um grande impacto na atratividade do investimento. Além do mais, como a eletricidade e o furfural têm praticamente a mesma participação na receita, flutuações em seus preços apresentam efeitos semelhantes na TIR.

Figura 3 - Sensibilidade da taxa interna de retorno do cenário $1 \mathrm{G}+\mathrm{FF}$

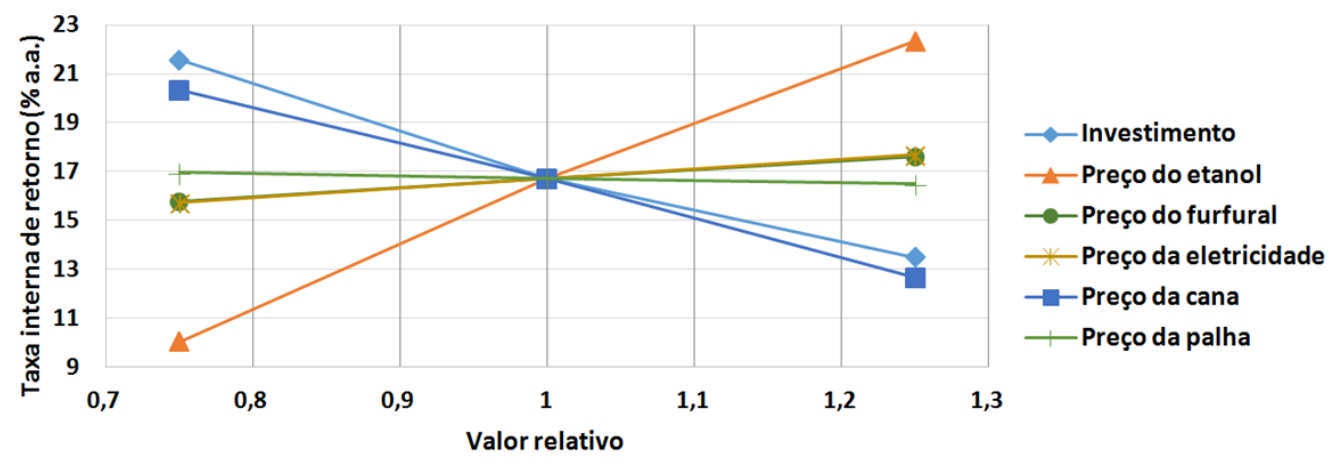




\section{CONCLUSÃO}

Neste trabalho, foi estudada a viabilidade da integração da produção de furfural via o processo contínuo Rosenlew à produção de etanol no contexto brasileiro. Dados de operação das plantas usados na simulação do processo refletem instalações industriais existentes. Os resultados obtidos mostraram que a integração da planta de furfural possui resultados extremamente positivos, uma vez que possibilita a obtenção de um produto de maior valor agregado. A taxa interna de retorno do investimento é de $16,7 \%$, significativamente maior que a da destilaria autônoma de etanol (12,7\%). Através da análise de sensibilidade, concluiu-se também que flutuações no preço do furfural não geram um grande impacto sobre a lucratividade do empreendimento, mostrando que a integração é realmente uma opção bastante atrativa.

\section{REFERÊNCIAS}

DIAS, M. O. S.; CUNHA, M. P.; JESUS, C. D. F.; ROCHA, G. J M.; PRADELLA, J. G. C.; ROSSELL, C. E. V.: MACIEL FILHO, R.; BONOMI, A.: Second-generation ethanol in Brazil: can it compete with electricity production? Bioresour. Technol., v. 102, 8964 8997, 2011.

DIAS, M. O. S.; JUNQUEIRA, T. L.; CAVALETT, O.; CUNHA, M. P.; JESUS, C. D. F.; ROSSELL, C. E. V.: MACIEL FILHO, R.; BONOMI, A.: Integrated versus stand-alone second generation etanol production from sugarcane bagasse and trash. Bioresour. Technol., v. 103, 152-161, 2012.

CTBE - Laboratório Nacional de Ciência e Tecnologia do Bioetanol: The Virtual Surgacane Biorefinery - 2011 Report. Relatório Técnico, 2012.

DE JONG, W.; MARCOTULLIO, G.: Overview of biorefineries on co-production of furfural, existing concepts and novel developments. Int. J. of Chem. Reactor Eng., v. 8, A69, 2010.

GVR: GRAND VIEW RESEARCH: Furfural market analysis by application (furfuryl alcohol, solvent) and segment forecasts to 2020. Relatório publicado por Grand View Research em janeiro de 2015.

MARIANO, A. P.; DIAS, M. O. S.; JUNQUEIRA, T. L.; CUNHA, M. P.; BONOMI, A.; MACIEL FILHO, R.: Utilization of pentoses from sugarcane biomass: technoeconomics of biogas vs. butanol production. Bioresor. Technol., v. 142, p. 390-9, 2013.

MOULIJN, J. A.; MAKKEE, M.; VAN DIEPEN, A. E.: Chemical Process Technology. John Wiler \& Sons: Chichester, Reino Unido. $2^{\mathrm{a}}$ ed., 2012.

ZEITSCH, K.J. The chemistry and technology of furfural and its many by-products. Sugar Series. Elsevier: Holanda, v. 13, p. 3, 48, 126, 304-306, 358-365, 2000. 